\title{
Chemical Interrogation of the Neuronal Kinome Using a Primary Cell- Based Screening Assay
}

\author{
Hassan Al-Ali, ${ }^{\dagger}$ Stephan C. Schürer, ${ }^{\ddagger}, \|$ Vance P. Lemmon, ${ }^{* \dagger, \ddagger, \S}$ and John L. Bixby ${ }^{* \dagger, \ddagger, \|}$ \\ ${ }^{\dagger}$ Miami Project to Cure Paralysis, ${ }^{\ddagger}$ Center for Computational Sciences, and Departments of ${ }^{\S}$ Neurological Surgery and ${ }^{\|}$Molecular \& \\ Cellular Pharmacology, University of Miami Miller School of Medicine, Miami, Florida 33136, United States
}

\section{Supporting Information}

ABSTRACT: A fundamental impediment to functional recovery from spinal cord injury (SCI) and traumatic brain injury is the lack of sufficient axonal regeneration in the adult central nervous system. There is thus a need to develop agents that can stimulate axon growth to re-establish severed connections. Given the critical role played by protein kinases in regulating axon growth and the potential for pharmacological intervention, small molecule protein kinase inhibitors present a promising therapeutic strategy. Here, we report a robust cell-based phenotypic assay, utilizing primary rat hippocampal neurons, for identifying small molecule kinase inhibitors that promote neurite growth. The assay is highly reliable and suitable for medium-throughput screening, as indicated by its $Z^{\prime}$-factor of 0.73 . A focused structurally diverse library of protein kinase inhibitors was screened, revealing several compound groups with the ability to strongly and

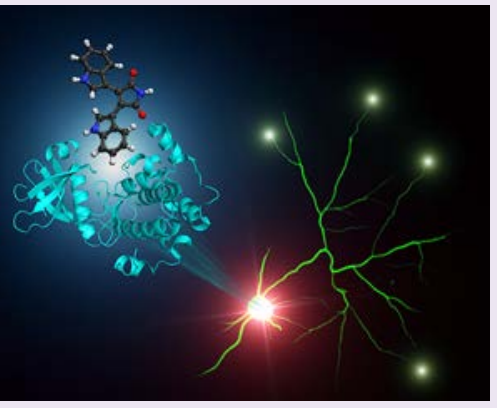
consistently promote neurite growth. The best performing bioassay hit robustly and consistently promoted axon growth in a postnatal cortical slice culture assay. This study can serve as a jumping-off point for structure activity relationship (SAR) and other drug discovery approaches toward the development of drugs for treating SCI and related neurological pathologies.

pinal cord injury (SCI) is estimated to affect more than 300,000 individuals in the U.S., with an additional 12,000 injuries occurring each year, creating an enormous medical, social, and economic burden on patients, their families, and the healthcare system. ${ }^{1}$ Failure to recover from SCI-associated loss of function is due, at least in part, to the lack of regenerative capacity in mature central nervous system (CNS) neurons. ${ }^{2}$ To date, no effective pharmacological treatment exists for enhancing axon regeneration following SCI. There is thus a pressing need to develop therapeutics that can promote axon regeneration and therefore SCI recovery.

Protein kinases are critical regulators of most cellular processes, granting them center stage in the search for new therapeutic drugs, most notably in cancer. ${ }^{3}$ In addition, drug discovery campaigns are utilizing protein kinases as targets in immunological, metabolic, and CNS disorders. ${ }^{3-8}$ A number of protein kinases have been implicated in controlling axonal growth and guidance in vitro and in vivo, ${ }^{9-12}$ making them attractive targets for pharmacological treatment of SCI. In vitro studies using high content analysis (HCA $)^{13}$ and large-scale in vivo screens have demonstrated that small molecule kinase inhibitors can indeed promote neurite growth. ${ }^{14}$

In recent years, the majority of first-in-class drugs have come from cell-based phenotypic screens, despite the greater focus on target-based screening. ${ }^{15}$ One problem with using small molecules for targeting protein kinases is the scarcity of selective protein kinase inhibitors (PKIs). Given the homology in the kinase domain of most protein kinases, the vast majority of competitive PKIs tend to have promiscuous activity profiles. ${ }^{16}$ Thus, target-based discovery of PKIs is plagued by the paucity of knowledge of their full activity profiles. Fortunately, there are PKIs with favorable selectivity profiles, and even some with multiple targets that contribute to therapeutic activity. ${ }^{17}$ It remains difficult, however, to identify such compounds using target-based screening, making phenotypic screening an attractive alternative for identifying PKIs with favorable physiological activity. Here, we report the development of a robust phenotypic HCA assay for identifying PKIs that promote neurite growth in primary neuronal cultures. We used this assay to screen a small but structurally diverse PKI library (InhibitorSelect Protein Kinase Inhibitor libraries I, II, and III from EMD Millipore). The best performing bioassay hit was tested in a follow-up study where it robustly and consistently promoted axon growth in a postnatal cortical slice culture assay.

\section{RESULTS AND DISCUSSION}

We developed a high content analysis (HCA) screening bioassay aimed both at identifying small-molecule PKIs that can promote neurite growth (Figure 1) and providing insight into the group of protein kinase targets that may be suitable for drug discovery in SCI. The assay utilizes a relevant cellular model, primary mammalian neurons, and analyses the effect of protein kinase inhibition on neurite morphology of these cells. We began by screening a small structurally diverse compound

\footnotetext{
Received: October 26, 2012

Accepted: March 12, 2013

Published: March 12, 2013
} 

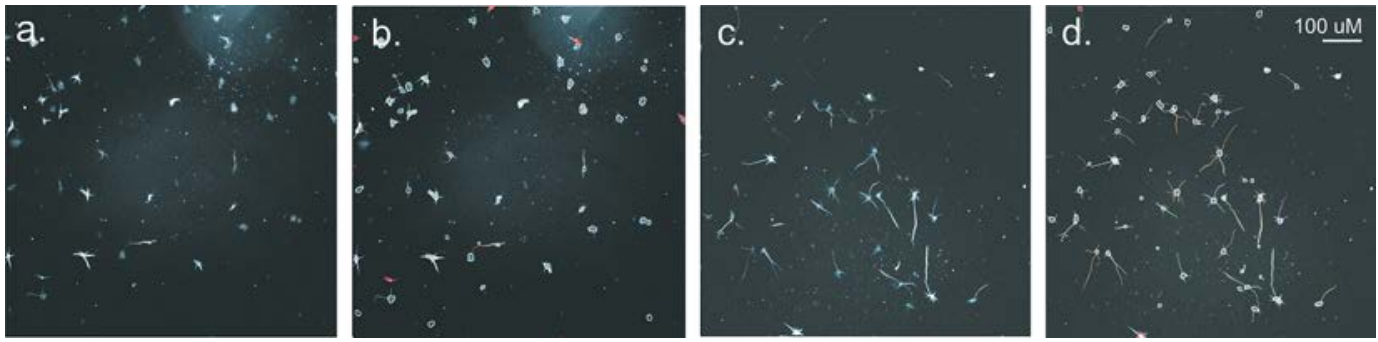

Figure 1. High content analysis of neurons in culture. Neurons in 96-well plates immunostained for $\beta$ III-tubulin (cell bodies and neurites) and nuclei (Hoechst). (a) DMSO-treated control cells, (b) traced image of panel a, (c) test compound-treated cells, (d) traced image of panel c. The images were automatically analyzed by the Neuronal Profiling Bioapplication software to yield dozens of morphological measurements for each neuron in the well.

set comprising 240 verified small-molecule PKIs (Supplementary Figure 1). Each compound was screened in duplicate at 6 different concentrations $(6 \mathrm{nM}, 32 \mathrm{nM}, 160 \mathrm{nM}, 800 \mathrm{nM}, 4$ $\mu \mathrm{M}$, and $20 \mu \mathrm{M})$. Data were acquired using a Cellomics Arrayscan VTI platform after neurons were fixed and immunostained for $\beta$ III-tubulin, allowing the acquisition of a readout of neurite morphology including length, count, and branching. Hits were defined as compounds that induced (at any of the tested concentrations) an average increase of neurite total length $(\mathrm{NTL}) \geq 25 \%$ relative to DMSO control, at nontoxic concentrations, and with a $Z$-score $>1.5$ in each of two independent experiments. These criteria yielded a low false discovery rate of $7 \%$, with an average $Z^{\prime}$-factor ${ }^{18}$ of 0.73 , indicating good reproducibility in independent screening experiments. Data points across the two screens had a Pearson coefficient $\left(r^{2}\right)$ of 0.81 . A validation screen performed one year later by independent personnel utilizing separate lots of all reagents (except PKI stocks) produced $87 \%$ agreement with the hits from the initial screen (Supplementary Table 1), further highlighting the robustness of the assay.

Neurite total length (NTL) was chosen as a first-pass filter to capture various processes that increase neurite growth, including increases in neurite count (NC), neurite average length (NAL), and neurite branch-point average (NBA). The majority of kinase inhibitor (KI) hits appeared to increase NTL mostly by increasing NAL, as is indicated by the high correlation between these two parameters (Figure 2A). The correlation between NAL and NBA was not strong, suggesting that neurite branching (measured by NBA) relies on different signals than neurite extension (measured by NAL). The possibility that these correlations may reflect target bias in the EMD library cannot be discounted; screening of larger, more diverse KI libraries will be required to obtain a more comprehensive view of the interrelationships among these attributes and their susceptibility to pharmacological perturbation.

Bioassay activity clustering (Figure 2B) shows that certain compounds, such as ML-7 and Flt-3 inhibitor III, had a broad effect on multiple aspects of neurite growth, increasing NAL, NBA, and NC. Others (e.g., VEGF Receptor tyrosine kinase inhibitor II) appeared to have more isolated effects that strictly influenced neurite length.

Active Hits and Scaffolds. The InhibitorSelect library (I, II, and III) constitutes a collection of structurally diverse, wellcharacterized, cell-permeable, mostly ATP-competitive inhibitors of kinases. The compounds were selected on the basis of having known inhibitory activity toward 50 well studied protein kinases and were thus grouped in panels that are proposed to assist mechanistic studies of signaling pathways. Examples of these pathways include the Akt/mTOR, EGFR, MAP Kinase, $\mathrm{NF}-\kappa \mathrm{B}, \mathrm{JAK} / \mathrm{STAT}, \mathrm{VEGF}, \mathrm{Wnt}$, IGF, and p53 signaling pathways. Consequently, the number of structurally related compounds within the library is too small for structure activity relationship (SAR) analyses in the case of most hits. However, there exist several groups of compounds with multiple active hits in our bioassay. These are likely to represent series with good SAR potential and will be discussed below. The complete list of all screened compounds is provided in Supplementary Table 2.

Isoquinolinyl Sulfonamides and ML-7. In addition to showing low toxicity, this class of compounds, together with their analogue ML-7, contains several compounds that were hits in our bioassay (Table 1). Compounds in this group exhibit inhibitory activity toward Rho kinase (ROCK) ${ }^{19}$ and include the drug Fasudil. Due to their effects on RhoA and/or myosin kinase, these compounds can modulate actin-myosin turnover and dynamics, two critical regulators of neurite growth. ${ }^{20-22}$ ROCK has been shown to mediate inhibition of neurite growth on inhibitory substrates such as myelin and/or chondroitin sulfate proteoglycans both in vitro and in vivo, ${ }^{23}$ and pharmacological inhibition or gene knockdown of ROCK restores normal neurite growth under growth inhibitory conditions. $^{12,24}$ Moreover, treatment with a ROCK inhibitor, $\mathrm{Y}-27632$, can induce CNS axon regeneration in vivo. ${ }^{25}$ Many ROCK inhibitors are isoform nonspecific; however, knockdown studies suggest that either ROCK1 or ROCK2 can mediate neurite growth inhibition. ${ }^{12}$ Of the five named ROCK inhibitors tested in this screen, four (Rho Kinase inhibitors I, II, IV, and V) induced sufficient neurite growth to qualify as hits. Rho Kinase inhibitor III, however, failed to induce neurite growth within the tested concentration range. This discrepancy may be explained by the fact that Rho Kinase inhibitor III has an $\mathrm{IC}_{50}$ of $25 \mu \mathrm{M}$ (higher than the highest tested concentration), while the other four all have $\mathrm{IC}_{50}$ 's in the nanomolar range. Interestingly, the more potent ROCK inhibitor, ROCK inhibitor $\mathrm{V}\left(\mathrm{IC}_{50}=1.5 \mathrm{nM}\right)$, had a lesser effect on promoting neurite growth than ROCK inhibitor IV $\left(\mathrm{IC}_{50}=11.8 \mathrm{nM}\right)$, despite having comparable or higher predicted cell permeability. This may indicate that off-target effects of ROCK inhibitor IV contribute to the compound's ability to promote neurite growth. Alternatively, off-target inhibition in the low micromolar to submicromolar range of kinases such as Akt, by ROCK inhibitor $\mathrm{V},{ }^{26}$ may counteract its neurite growth-promoting activity. Studies have shown that Akt activity promotes, and may be required for, neurite elongation. ${ }^{27}$ This is not surprising, given that Akt is an upstream activator of $\mathrm{mTOR}$, a positive regulator of neurite growth. $^{11,28,29}$ 

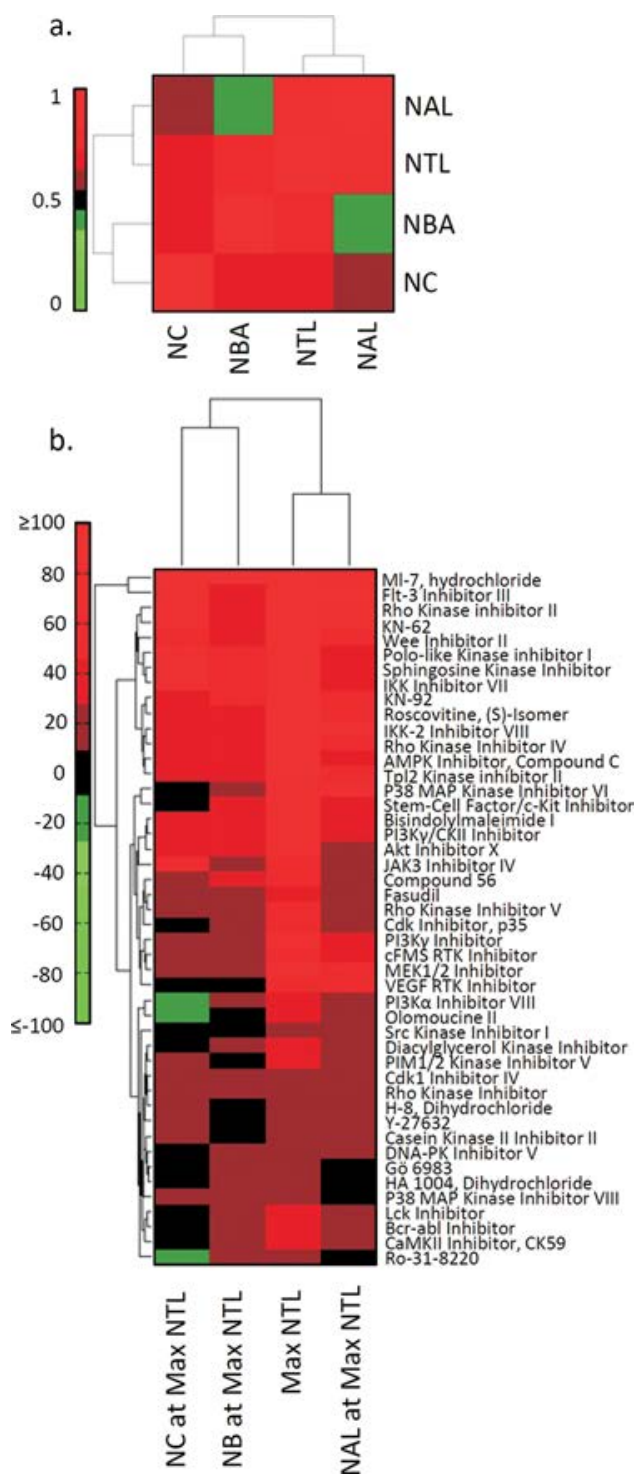

Figure 2. Effects of hit compounds on distinct neurite parameters. (a) The Pearson correlation coefficient $\left(r^{2}\right)$ was calculated for each pair of neurite features using the data obtained for each hit $\mathrm{KI}$ at the concentration at which maximum neurite total length (NTL) was observed. (b) The effects (\% change from control) of KI hits on NTL, NAL, NBA, and NC are shown at the concentrations at which maximal effect on NTL was observed for each compound. Clustering was performed in MATLAB using a Euclidean metric in the KI dimension and a correlation metric in the neurite feature dimension. Activity clustering shows that certain compounds, such as ML-7 and Flt-3 inhibitor III, increased multiple aspects of neurite growth, while others, such as VEGF Receptor tyrosine kinase inhibitor II, appeared to have more isolated effects on neurite length.

The isoquinoline 5-sulfonyl analogue ML-7 had the most pronounced effect on neurite length increase. Neurite length in ML-7 treated cells almost tripled in comparison to controls, with an $\mathrm{EC}_{50}$ of about $74 \mathrm{nM}$. There is currently no information on the activity of ML-7 toward ROCK, although multiple accounts suggest that ROCK is not a target of ML-7. ${ }^{30}$ Moreover, ML-7's maximum effective concentration in our bioassay is orders of magnitude lower than that of ROCK inhibitor IV, suggesting a distinct mechanism of action that is less likely to be related to ROCK inhibition, and more likely to be associated with a specific target of ML-7. MLCK, the primary target of ML-7, has been shown to play an important role in controlling growth cone dynamics and axon growth. In fact, MLCK and ROCK each appear to inhibit neurite growth, at least in part, by controlling phosphorylation of myosin light chain (MLC). ${ }^{31}$ MLCK phosphorylates MLC, while ROCK inhibits the phosphatase that reverses the phosphorylation, with both events leading to neurite growth inhibition.

Bisindolylmaleimides. Bisindolylmaleimides are potent inhibitors of protein kinase C (PKC). PKC has been implicated in mediating inhibitory signaling by chondroitin sulfate proteoglycans (CSPGs), and inhibition of PKC activity promoted axon regeneration in a rat spinal cord injury model. ${ }^{32}$ Paradoxically, PKC has previously been shown to play a positive role in neurite growth. ${ }^{33}$ The disparity in PKC's involvement is likely to be due to different isoforms or paralogs operating within distinct signaling contexts. Expectedly, bisindolylmaleimides exhibit differential activity toward different PKC isoforms. ${ }^{19}$ Additionally, they are known to be inhibitors of glycogen synthase kinase-3 activity, another effector of neurite growth. ${ }^{11}$ Activity profiling shows that Binsindolylmaleimide I has inhibitory activity toward ribosomal S6 kinases (RSKs). RSKs belong to the family of AGC kinases and have been shown to negatively regulate axon growth in motoneurons. ${ }^{34}$ Compared to bisindolylmaleimide IV, bisindolylmaleimide I and Gö 6983 show higher activity toward PKC, GSK, and RSK, ${ }^{19}$ which might explain why bisindolylmaleimide IV failed to produce a hit in our bioassay (Table 2).

Anilinoquinazolines. 4-Anilinoquinazoles constitute an important class of ATP-competitive tyrosine kinase inhibitors. Substitution with a bulky analogue at the 4 '-position of the anilino group has been shown to make a compound both ATP competitive and peptide competitive. This is due to steric clash between the bulky group and the substrate tyrosine residue. Indeed, these compounds show inhibitory activity toward ABL1/2, ACK1, BRK, c-MER, c-SRC, LYN, EGFR, ERBB2, ERBB4, FGR, and FLT3 protein tyrosine kinases and some serine/threonine kinases such as STK10 and MNK2. ${ }^{19}$ Several studies have implicated protein tyrosine kinases in regulating axon growth, ${ }^{12,35}$ and it has long been known that protein tyrosine kinase inhibitors can promote axon growth. ${ }^{35}$ An siRNA knockdown screen using cerebellar granule neurons associated downregulation of ERBB2, FGR, and FLT3 with reduced neurite growth, while downregulation of EGFR was associated with improved growth over inhibitory substrate. ${ }^{12}$ Src kinase inhibitor I shows the highest activity toward HCK, ${ }^{19}$ whose knockdown was also associated with improved neurite growth. ${ }^{12}$ Multiple compounds within this group show a marginal effect on neurite growth-promotion, but only two, Src Kinase Inhibitor I and Compound 56, pass the hit classification filter (Table 3).

Diaminopurines. The InhibitorSelect library contains 15 compounds possessing the 2,6-diamino-purine framework, most of which are substituted at the 2, 6, and 9 positions (Table 4). Many of these compounds are CDK inhibitors and show activity toward CDK1, CDK2, CDK3, and CDK5. ${ }^{19}$ In this group, Olomoucine II displays increased potency toward $\mathrm{CDK} 1 / \mathrm{B}\left(\mathrm{IC}_{50}=20 \mathrm{nM}\right)$ compared to Roscovitine $\left(\mathrm{IC}_{50}=450\right.$ $\mathrm{nM})$ or Bohemine $\left(\mathrm{IC}_{50}=1.1 \mu \mathrm{M}\right)$. Interestingly, Olomoucine II exhibited lower cytotoxicity in primary hippocampal neurons than was observed in various cancer cell lines, possibly because primary neurons are nondividing. It is currently unclear whether these compounds promote neurite growth in hippocampal neurons by directly inhibiting CDK activity. Enzymatic 
Table 1. Isoquinolinyl Sulfonamides and ML-7

Isoquinolinyl sulfonamides

$[\mathrm{C}, \mathrm{N}]$<smiles></smiles>

ML-7

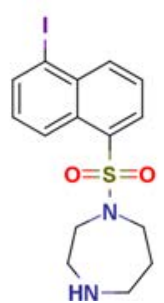

Rho Kinase Inhibitor IV

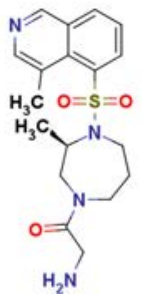

Fasudil

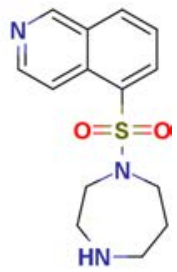

\begin{tabular}{llcc}
\hline \multicolumn{1}{c}{ compound name } & CAS no. & concn at maximal effect & max NTL effect (\%) \\
ML-7, Hydrochloride & $110448-33-4$ & $160 \mathrm{nM}$ & 288 \\
Rho Kinase Inhibitor IV & $913844-45-8$ & $20 \mu \mathrm{M}$ & 277 \\
HA1077, Fasudil & $103745-39-7$ & $20 \mu \mathrm{M}$ & 176 \\
H-8 & $113276-94-1$ & $20 \mu \mathrm{M}$ & 149 \\
HA 1004 & $92564-34-6$ & $20 \mu \mathrm{M}$ & 149 \\
H-89 & $127243-85-0$ & inactive & NA \\
Rho Kinase Inhibitor & not registered & inactive & NA
\end{tabular}

Table 2. Bisindolylmaleimides

Bisindolylmaleimides<smiles>[R]N1C(=O)C(c2cn([R])c3ccccc23)=C(c2cn([R])c3ccccc23)C1=O</smiles>

Bisindolylmaleimide I

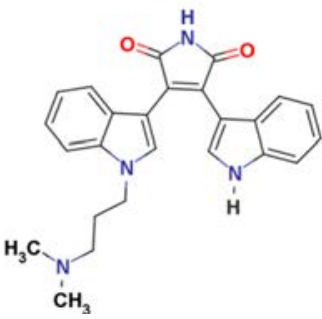

Gö 6983

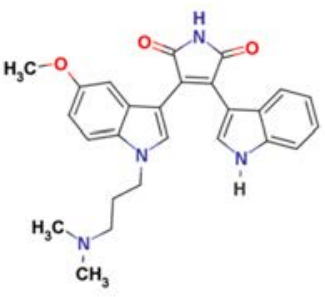

concn at maximal effect

$800 \mathrm{nM}$

$4 \mu \mathrm{M}$

$800 \mathrm{nM}$

inactive

inactive

inactive
Bisindolylmaleimide V

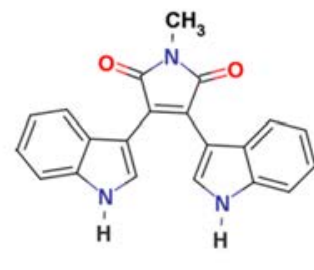

$\max$ NTL effect (\%)

183

144

127

NA

NA

NA
119139-23-0

$683775-59-9$ profiling shows that the compounds also have inhibitory activity toward various protein tyrosine kinases, such as SRC, FYN, and FLT3. $^{19}$

Effect of PKI Hits on the Activation of Neurite Growth Signaling Markers. Cell signaling pathways constitute complex, robust, and often redundant networks. Protein kinases regulate the operation of multiple effectors in various signaling pathways to control neurite growth and axon regeneration. ${ }^{9,10,12}$ It is thus possible that the various PKI hits affect neurite growth by modulating different signaling pathways in distinct manners. To examine this, we probed the phosphorylation status of six downstream effectors of signaling pathways previously associated with neurite growth or inhibition (Supplementary Figure 2): ribosomal RPS6, ${ }^{11}$ GSK3 $\beta{ }^{36}$ Cofilin- $1,{ }^{37} \mathrm{NF} \kappa$-B, ${ }^{38}$ c-Jun, ${ }^{39}$ and P42/44 MAPK. ${ }^{11}$ Relevant phosphorylation sites were probed by Western blotting following treatment of HEK293T cells with 12 of our PKI hits. The 12 compounds were selected to represent the different scaffolds of the most active PKI hits (Figure 3).

As shown in Figure 4, the tested PKIs affect the phosphorylation of the various markers both positively and negatively. Hierarchical clustering demonstrates the presence of broad similarities in behavior among some of the PKIs, suggesting commonalities of signal pathway perturbation. As predicted, however, there is no denominating fingerprint for all growth stimulators. A follow-up study is necessary to analyze the mechanisms of action of these hits and explore the potential for improving neurite growth by simultaneously targeting multiple growth-inhibitory kinases.

Non-protein Kinase Inhibitors. The screened library also contained a small number of non-protein kinase inhibitors, some of which were able to consistently promote neurite growth in our bioassay. Several PI3K inhibitors showed neurite growth-promoting activity. Interestingly, several PI3K inhibitors were able to promote neurite growth despite having unrelated structures, providing convergent lines of evidence for a direct role for PI3K, or a homologous kinase, in influencing neurite length. Some of these inhibitors show isoform selectivity toward $\mathrm{PI} 3 \mathrm{~K} \alpha, \mathrm{PI} 3 \mathrm{~K} \beta$, and $\mathrm{PI} 3 \mathrm{~K} \gamma$, though there does not seem to be any clear preference for inhibition of either isoform in neurite growth-promotion. PI3K is an upstream effector of the Akt/mTOR pathway, which appears to be intimately associated with axon growth and regeneration. However, previous reports suggest that PI3K activity is a positive regulator of neurite growth. ${ }^{11,28}$ As expected, treatment with selective mTOR inhibitors inhibited neurite growth in our 
Table 3. Anilinoquinazolines

Anilinoquinazolines

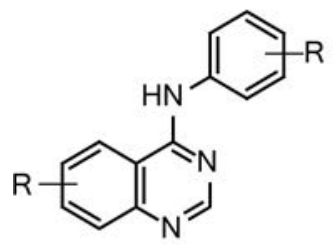

Src Kinase Inhibitor I

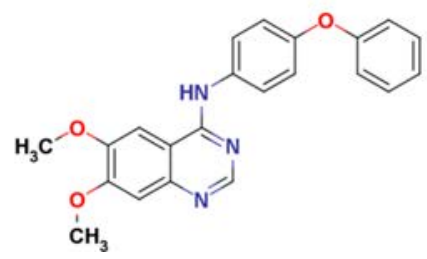

Compound 56<smiles>CCOc1cc2ncnc(Nc3cccc(Br)c3)c2cc1OCC</smiles>

\begin{tabular}{lccc}
\hline \multicolumn{1}{c}{ compound name } & CAS no. & concn at maximal effect & max NTL effect (\%) \\
Src Kinase Inhibitor 1 & $179248-59-0$ & $20 \mu \mathrm{M}$ & 174 \\
Compound 56 & $171745-13-4$ & $20 \mu \mathrm{M}$ & 142 \\
BPIQ-I & $174709-30-9$ & inactive & NA \\
PD 158780 & $171179-06-9$ & NA \\
PD 174265 & $216163-53-0$ & inactive & NA \\
Aurora Kinase Inhibitor II & $331770-21-9$ & inactive & NA \\
EGFR/ErbB-2 Inhibitor & $179248-61-4$ & inactive & NA \\
JAK3 Inhibitor II & $211555-04-3$ & inactive & NA \\
PDGF Inhibitor III & $205254-94-0$ & inactive & NA \\
AG 1478 & $175178-82-2$ & inactive & NA \\
Ste11 MAPKKK Inhibitor & $331662-51-2$ & inactive & NA \\
WHI-P180, Hydrochloride & $211555-08-7$ & inactive & NA \\
\hline
\end{tabular}

Table 4. Diaminopurines

Diaminopurines<smiles>[R]Nc1nc(N[R])c2ncn([R])c2n1</smiles>

Olomoucin II

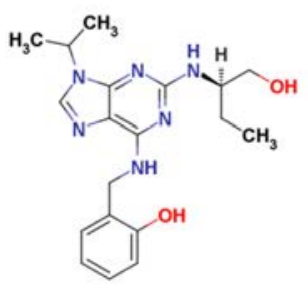

Roscovitine, (S)-Isomer

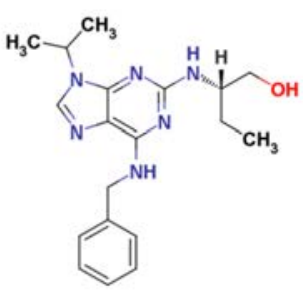

CaMKII Inhibitor, CK59

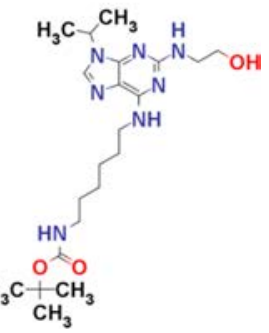

max NTL effect (\%) concn at maximal effect

$4 \mu \mathrm{M}$

$20 \mu \mathrm{M}$

$20 \mu \mathrm{M}$

$20 \mu \mathrm{M}$

inactive

inactive

inactive

inactive

inactive

inactive

inactive

inactive

inactive

inactive

inactive
138

193

164

159

NA

NA

NA

NA

NA

NA

NA

NA

NA

NA

NA assay (data not shown). The growth promoting activity of the PI3K inhibitors may therefore be due to unintended targets. PI3Ks are members of the PI3/PI4-kinase family, which includes mTOR, DNA-PK, ATM, ATR, and PI4K. Structural similarity among these kinases can lead to promiscuity among PI3K inhibitors. Interestingly, an inhibitor of another member of this family, DNA-PK Inhibitor V, was also found to induce neurite growth, albeit weakly and at the highest tested concentration. It is also possible that the off-target effect is mediated by kinases unrelated to the PI3/PI4-kinase family. For example, PI3-K $\gamma$ inhibitor also inhibits $\mathrm{MUSK}^{19}$ a tyrosine receptor kinase shown to be involved in inhibiting neurite growth. ${ }^{40}$

Another non-protein kinase inhibitor that was able to promote neurite growth is Sphingosine Kinase Inhibitor. In a comprehensive activity profiling panel, this compound showed no activity toward any of 250 assayed protein kinases, ${ }^{19}$ highlighting its unusual specificity. Sphingolipids serve as important second messengers in multiple cellular events ranging from growth to survival and differentiation. ${ }^{41}$ Recently, 


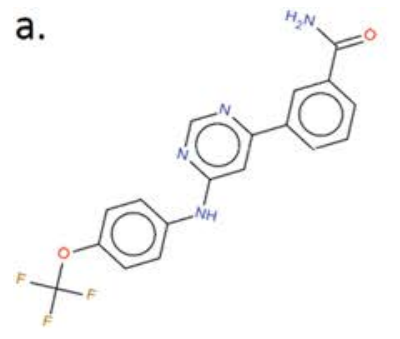

b.

f.

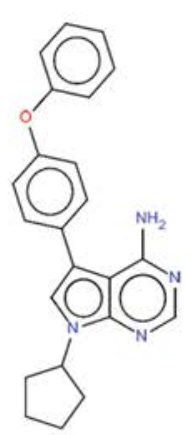

i.
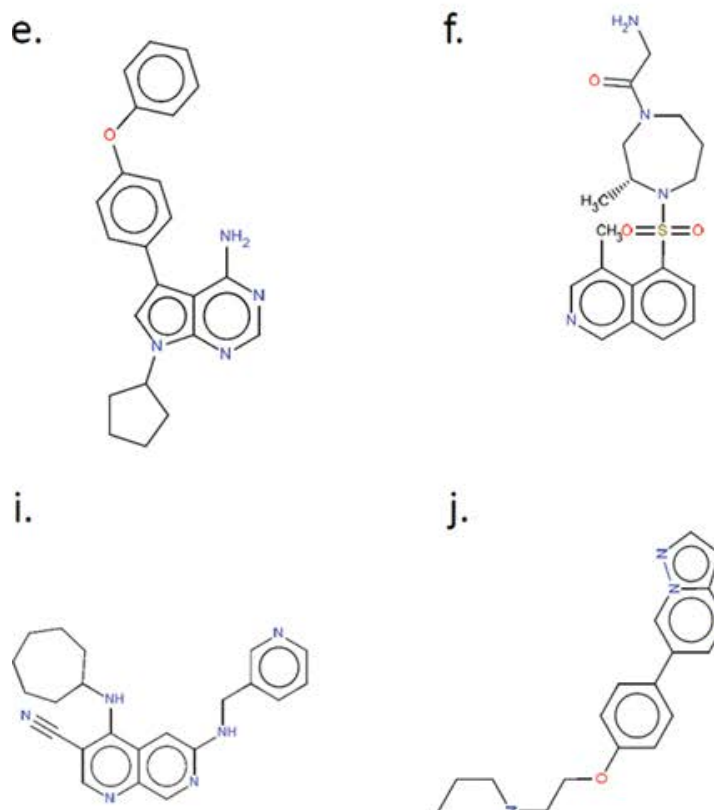

j.
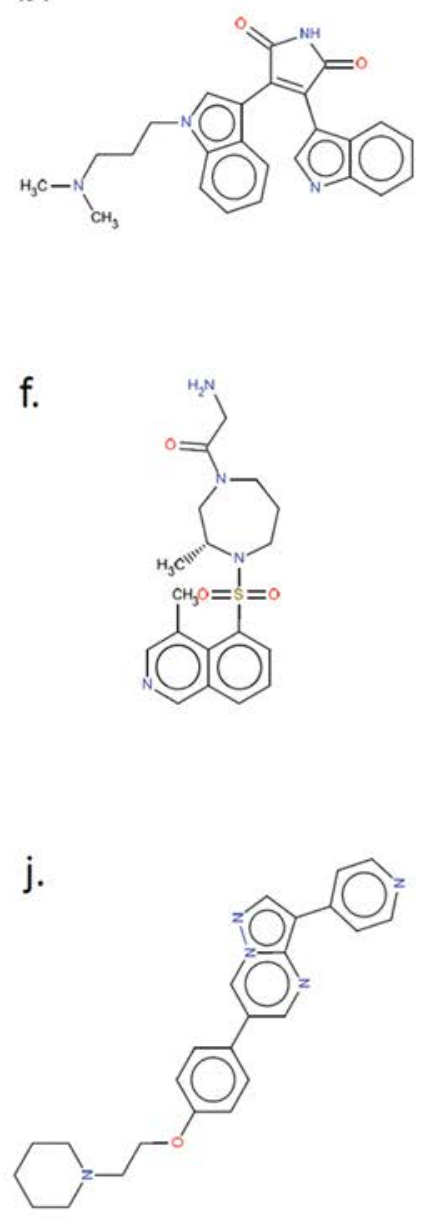

c.<smiles>CCOc1cc2ncnc(Nc3cccc(Br)c3)c2cc1OCC</smiles>

g.

d.

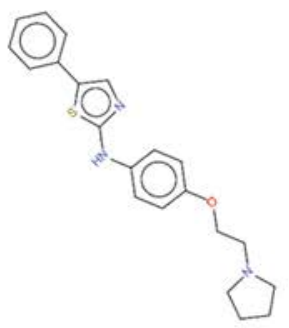

h.
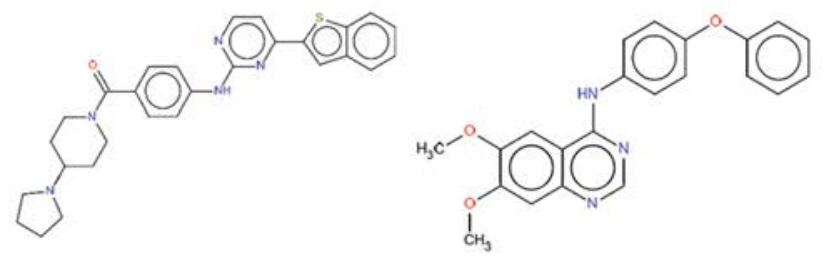

k.

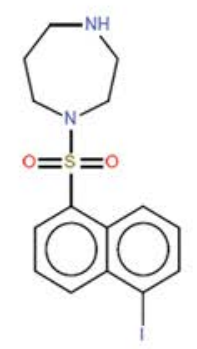

I.

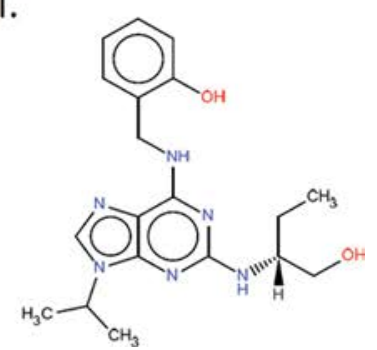

Figure 3. 12 PKI hits representing different scaffolds and structures: (a) Bcr-abl Inhibitor, (b) bisindolylmaleimide I, (c) Compound 56, (d) Flt-3 Inhibitor III, (e) Lck Inhibitor, (f) Rho Kinase Inhibitor IV, (g) IKK Inhibitor VII, (h) Src Kinase Inhibitor I, (i) Tpl2 Kinase Inhibitor II, (j) AMPK inhibitor, (k) ML-7, and (1) Olomoucine II.

sphingosine-1-phosphate (S1P) has been shown to play a role in neuronal differentiation and neurite growth. ${ }^{42}$ Extracellular S1P negatively influences axon growth and induces retraction, possibly via a Rho-dependent mechanism. ${ }^{43}$ It is unclear whether Sphingosine Kinase Inhibitor is promoting neurite growth by limiting extracellular levels of S1P or by indirectly influencing related pathways via alterations in endogenous levels of sphingosine or downstream metabolites such as ceramide.

Bioassay Hit Promotes Axon Growth in Postnatal Cortical Neurons. To test whether a hit PKI identified in our cell-based bioassay can promote axon growth in an environment that better recapitulates CNS conditions, we investigated the neurite growth activity of ML-7, our best performing bioassay hit, in organotypic slice cultures. ${ }^{44}$ This cortical slice assay can be used as a secondary screen to identify promising hits before proceeding to in vivo testing. Bilateral slices of P5 cortices were labeled with AAV8 viral particles expressing EGFP (AAV8-EGFP), then transected (after 8 DIV) at the midline, and cultured in apposition to an unlabeled contralateral half slice (Figure 5a). Paired slices received either ML-7 $(10 \mu \mathrm{M})$ or DMSO alone in their culture media and were incubated for 7 days to allow labeled axons to grow into the juxtaposed contralateral slice. After fixation, confocal microscopy was used to image a strip of the unlabeled slice $1 \mathrm{~mm}$ away from the apposition site, and confocal images were stacked and combined into a single image for quantification. Automated tracing was performed using the neurite detection algorithm developed by Wu et al. ${ }^{45}$ as implemented in MatLab (Figure 5a-c). Treatment with ML-7 induced a visible increase in the number of labeled axons crossing into the unlabeled slice (Figure $5 \mathrm{~b}$ ), and quantification revealed that, compared to a DMSO control, slices receiving ML-7 had significantly more crossed axons (Figure 5d). It is thus possible to identify with our PKI screen compounds that promote axon growth in an organotypic context.

Potential for Targeted Drug Discovery. We identified over 40 kinase inhibitors that were able to cross the hit classification threshold, and a number of scaffolds that were enriched within active compounds (Supplementary Table 3). While the exact mechanisms of action of these compounds and their corresponding targets remain to be elucidated, this study provides an informative starting point for the development of PKIs with better neurite growth-promotion activity and toxicity profiles. Several kinases are potential targets for growth promoting PKIs based on their frequency and extent of inhibition by PKI hits in our screen (Supplementary Table 3 and ref 19). These include ROCKs, PKCs, CDKs, and RSKs. Knockdown studies using shRNA will elucidate which of these kinases, when downregulated, can lead to increased neurite 

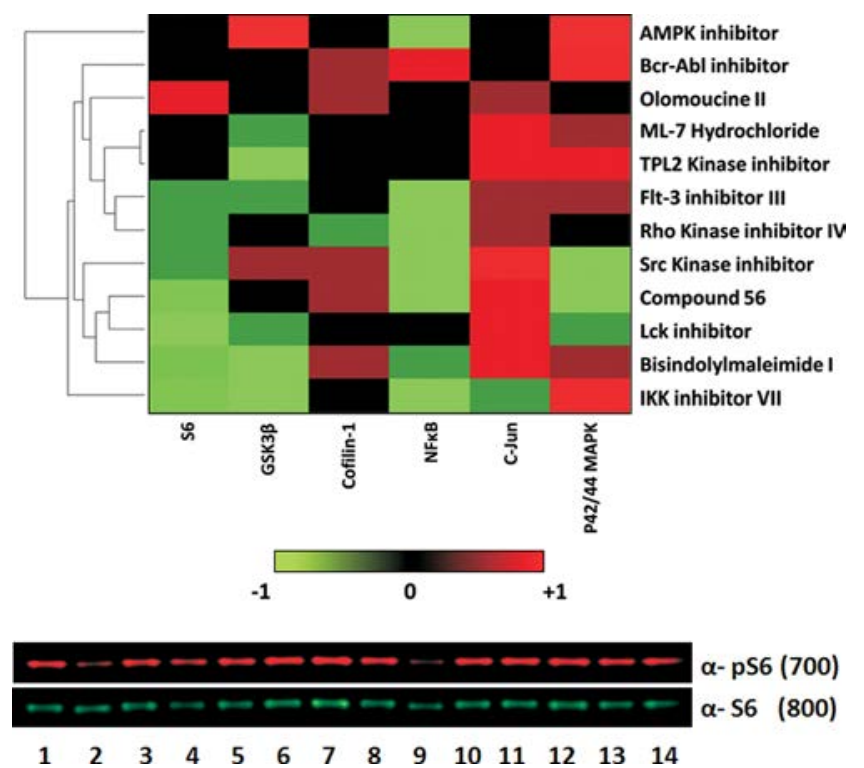

Figure 4. Densitometric analysis of phosphorylation by Western blotting: Phosphorylation of indicated markers was analyzed by Western blotting in HEK 293T cells treated with 12 different PKI hits for $3 \mathrm{~h}$. Each colored block represents the change of phosphorylation, where black means no change, red means increased phosphorylation, and green means decreased phosphorylation (values are averaged over three independent experiments). Hierarchical clustering was performed in MatLab using a Euclidean metric. A sample blot is included for total S6 ( $\alpha$-S6) and phospho-S6 ( $\alpha$-pS6): (1) Bcr-abl inhibitor, (2) bisindolylmaleimide I, (3) Compound 56, (4) Flt-3 inhibitor III, (5) Lck inhibitor, (6) ROCK inhibitor IV, (7) Src Kinase inhibitor I, (8) AMPK inhibitor, Compound C, (9) IKK inhibitor VII, (10) ML-7, Hydrochloride, (11) Olomoucine II, (12) Tpl2 Kinase inhibitor II, (13 and 14) DMSO (solvent). Representative Western blots for all markers are provided in Supplementary Figure 3.

growth and can help validate specific isoforms as potential drug targets for alleviating regenerative failure in the injured spinal cord.

This study serves as a proof of principle that a phenotypic screen of PKIs, in combination with knowledge of the activities of these compounds on cellular protein kinases, can be used to identify suitable drug targets. Additional screening of larger libraries spanning more chemical space and with better kinome coverage, along with comprehensive kinase activity profiling of hit and some non-hit PKIs, will lead to more accurate identification of target (as well as anti-target) kinases.

\section{METHODS}

Poly-D-lysine (P7886-500MG) was purchased from Sigma Aldrich (St. Louis, MO). Mouse anti- $\beta$ III-tubulin antibody was prepared inhouse, ${ }^{46}$ and Alexa Fluor 488 cross-linked goat anti-mouse antibody (A11029) was purchased from Life Technologies (Gaithersburg, MD). Primary antibodies $\alpha$-S6 (2317), $\alpha$-GSK3 $\beta$ (9832), $\alpha$-cJun (3270), $\alpha$ Cofilin-1 (5175), $\alpha$-P42/44 MAPK (9107), $\alpha$-NF- $\kappa \mathrm{B}$ (6956), $\alpha$ pSer240/244 S6 (2215), $\alpha$-pSer9 GSK3 $\beta$ (5558), $\alpha$-pSer73 c-Jun (2315), $\alpha$-pThr202/Tyr204 P42/44 MAPK (4370), and $\alpha$-pSer276 NF- $\kappa$ B (3031) were purchased from Cell Signaling (Danvers, MA), and $\alpha$-pSer3 Cofilin-1 (sc-271923) was purchased from Santa Cruz Biotechnology (Santa Cruz, CA). Secondary antibodies $\alpha$-mouse[IR800] (926-32210) and $\alpha$-mouse-[IR680] (926-32220) were purchased from LiCor, while $\alpha$-rabbit-[IR800] (611-732-127) and $\alpha$ rabbit-[IR680] (611-130-002) were purchased from Rockland (Gilbertsville, PA). Hippocampal tissue was incubated in HibernateE from BrainBits (Springfield, IL), supplemented with NeuroCult SM1
(05711) from StemCell Technologies (Vancouver, Canada). Cells were cultured in NbActive4 media from BrainBits. InhibitorSelect Protein Kinase Inhibitor libraries I, II, and III (approximately 240 compounds) were purchased from EMD Millipore (Billerica, MA). All other reagents were purchased from Life Technologies.

Screening Assay. We have developed a HCA screening assay for identifying PKIs that promote neurite growth in primary neuronal cultures. The assay utilizes dissociated rat hippocampal neurons, obtained from rat embryos (E18) as previously described. ${ }^{10}$ Dissociated cells were immediately seeded in 96-well plates (previously coated overnight with PDL at $0.5 \mathrm{mg} \mathrm{mL}^{-1}$ in HBSS and washed 5 times with PBS) at 1000 cells per well in $150 \mu \mathrm{L}$ of NbActive 4 and allowed to adhere for $2 \mathrm{~h}$ before PKI treatment. Treatment wells (duplicates) received compounds, prepared in DMSO, at final concentrations of $6 \mathrm{nM}, 32 \mathrm{nM}, 160 \mathrm{nM}, 800 \mathrm{nM}$, $4 \mu \mathrm{M}$, and $20 \mu \mathrm{M}$. Control wells (12 per plate) received DMSO solvent. The maximum final concentration of DMSO in control and treatment wells was $0.2 \% \mathrm{v} / \mathrm{v}$. DMSO at this concentration had no effect on any of the investigated neuronal parameters (data not shown). Cells were incubated with PKIs for $48 \mathrm{~h}$, after which the plates were fixed with $4 \%$ paraformaldehyde/ $4 \%$ sucrose in PBS at RT for $30-45 \mathrm{~min}$, rinsed with PBS, and stained for $\beta$ III-tubulin and nuclei (Hoechst dye).

Imaging and Tracing. Plates were imaged using a Cellomics ArrayScan VTI in 2 different channels for nuclear staining (Hoechst) and cell body/neurite staining ( $\beta$ III-tubulin). Nine fields per well were imaged with a $5 \mathrm{X}$ objective and automatically traced by the Neuronal Profiling Bioapplication version 3.5. Between 200 and 400 cells were measured per condition.

Data Acquisition and Analysis. Raw data were managed by the Cellomics Store, which consists of an SQL database and a networkattached fileserver (HP). Data for all traced cells and the values for all the catalogued attributes (total neurite length, cell body shape, cell body size, nuclear intensity, etc) were exported from the SQL server in spreadsheet format. Subsequent data processing was automated in MatLab. Several filters were applied to eliminate artifacts from entries using empirically determined cutoffs. For example, a minimum total neurite length of $10 \mu \mathrm{m}$ was set for detected cells, to avoid inclusion of dead cells and debris when calculating total average neurite length. Additional filters include attributes for cell body size $\left(<1800 \mu \mathrm{m}^{2}\right)$, number of neurite branches $(<24)$, and maximum neurite length without branches $(<800 \mu \mathrm{m})$. After all filters were applied, the remaining objects constituted valid neurons. Processed data from valid neurons were finally analyzed to detect neurite growth-promoters. These were defined as compounds that induced an average relative increase of neurite total length (NTL) of $25 \%$ or higher, at nontoxic concentrations, in 2 independent experiments and with a $Z$ score $>1.5$ in both experiments. The assay $Z^{\prime}$-factor ${ }^{18}$ was calculated using ML-7 as a control for neurite growth promotion and Torin-2 as a control for neurite growth inhibition.

Toxicity Assessment. To distinguish live cells (with larger dimly stained nuclei) from dead cells (with smaller brightly stained condensed nuclei), the 2D plot of nuclear total intensity versus nuclear total area of the traced cells within each plate was fitted to a two-component Gaussian mixture distribution. Viability in each well was computed as the fraction of live cells over the total cell count. For each culture plate, the viability in each well $\left(x_{t}\right)$ was transformed into change relative to the median of the pool $\left(x_{c}\right)$ of DMSO control wells $\left(r x=\left(x_{t}-x_{c}\right) / x_{c}\right)$. The standard deviation $\left(s d_{c}\right)$ of the pool of the DMSO controls (12 wells per plate) was used to transform relative changes into $\mathrm{Z}$ scores $\left(Z=x_{t}-x_{c} / s d_{c}\right)$. A treatment condition was considered toxic if it caused viability to fall below $60 \%$ with $Z$ score < -3 in the two independent screens.

Western Blot Analysis of Phosphorylation of Neurite Growth Signaling Markers. HEK293T cells were cultured in DMEM (Gibco) medium containing 10\% FBS and supplemented with penicillin/streptomycin. Cells were split into 3 cultures and propagated for 1 day before treatment with PKIs. Cells were seeded into 24-well plates at $0.25 \times 10^{6}$ cells per well in $0.5 \mathrm{~mL}$ of medium. PKI stock solutions (10 mM in DMSO) were diluted in cell culture 
a.

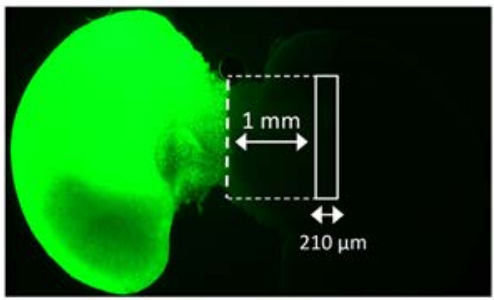

d.

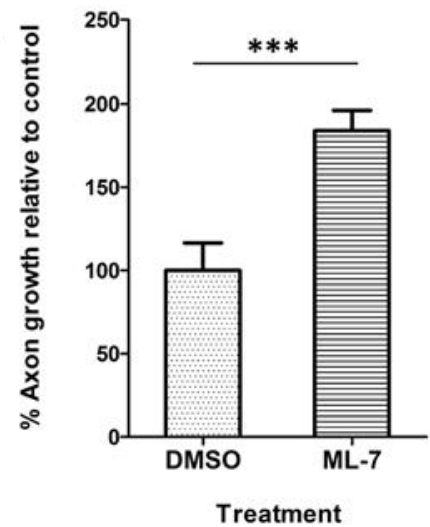

b.

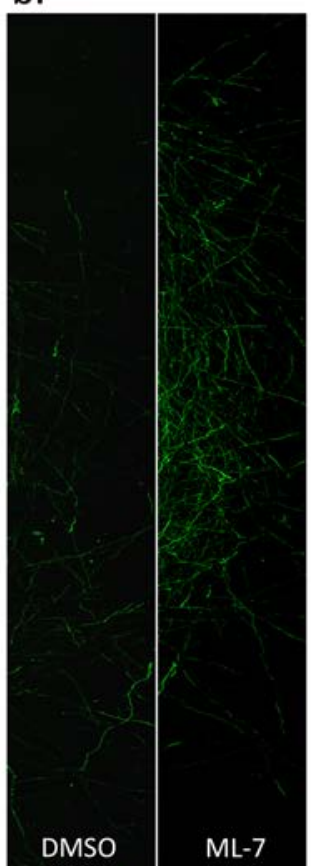

C.

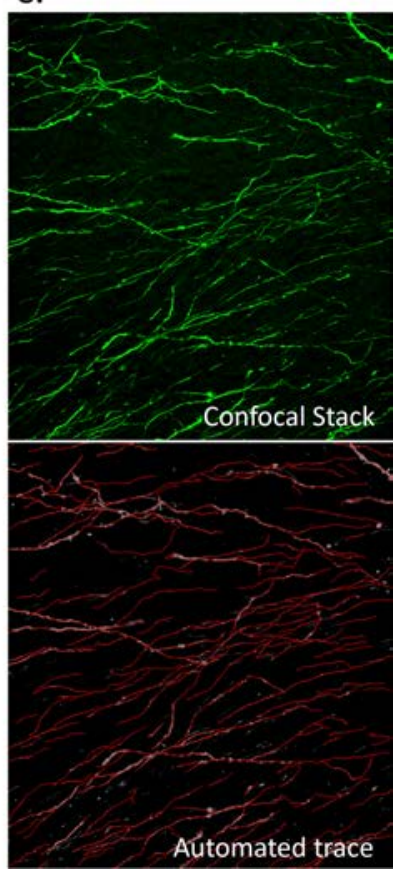

Figure 5. ML-7 promotes axon growth in transected cortical slice cultures. (a) Bilateral cortical slices connected by the corpus callosum were prepared from P5 rats and treated with viral particles encoding eGFP. After 8 DIV, the hemispheres were separated, paired with unlabeled P5 recipient tissue, and treated with ML-7 $(10 \mu \mathrm{M})$ or DMSO (control). Axon growth was evaluated 7 days later at a distance of $1 \mathrm{~mm}$ from the pairing interface. (b) Confocal Z stack of image strips obtained from a slice that received DMSO and a slice that received ML-7. (c) Sample of traced image using automated tracing implemented in MatLab (top: confocal image, bottom: gray scale version of confocal image with superimposition of traced axons in red). (d) Three replicate slices from two experiments were analyzed. $* * * P<0.01$, paired $t$ test, mean \pm SEM.

medium to a final concentration of $10 \mu \mathrm{M}$. Each well received $0.5 \mathrm{~mL}$ of the corresponding PKI solution, bringing the final concentration to $5 \mu \mathrm{M}$. Control wells received the corresponding volume of DMSO $(0.05 \%)$ alone. Each plate contained two DMSO control wells. Three hours following administration of compounds, the medium was aspirated and cells were washed once with PBS. The monolayer was then scraped into $250 \mu \mathrm{L}$ of hot SDS loading buffer (preheated in a boiling water bath) containing protease and phosphatase inhibitors. Samples were immediately placed in a heat block and kept at $95^{\circ} \mathrm{C}$ for $10 \mathrm{~min}$. Finally, samples were cooled on ice and then stored at $-80^{\circ} \mathrm{C}$ until needed (no longer than 2 weeks). Analytical SDS-PAGE was performed using precast NuPAGE Novex 4-12\% Bis-Tris 15-well gels (Invitrogen) run at $150 \mathrm{~V}$ (constant) in MOPS running buffer for $1 \mathrm{~h}$ or until the tracking dye reached the bottom of the slab. Protein was transferred from the gel to a nitrocellulose membrane in a wet transfer apparatus using $25 \mathrm{mM}$ sodium bicarbonate as transferring solution. Membranes were blocked with Odyssey blocking buffer for at least 1 hour and then incubated overnight in primary antibody solution (1:1 PBS, Odyssey blocking buffer with $0.1 \%$ Tween-20) at $4{ }^{\circ} \mathrm{C}$ on a shaker. Membranes were washed $3 \mathrm{x}$ in PBS-Tween-20 (10 min washes) and incubated in secondary antibody solution (secondary antibodies in 1:1 PBS, Odyssey blocking buffer with $0.1 \%$ Tween-20 and $0.02 \%$ SDS) at RT for $2 \mathrm{~h}$ with shaking. 800-IR-dye conjugated secondary antibodies were used to develop total protein bands, and 680-IR-dye conjugated secondary antibodies were used to develop phosphorylated protein bands. Finally, membranes were washed $5 \mathrm{x}$ in PBS Tween-20 (10 min washes). Blots were scanned using an Odyssey system. Relative change in phosphorylation in response to treatment was computed as

$$
\text { change in phosphorylation }=\frac{\frac{\text { normalized sample } 700 \text { signal }}{\text { normalized sample } 800 \text { signal }}}{\frac{\text { normalized control 700 signal }}{\text { normalized control } 800 \text { signal }}}
$$

Calculated values were averaged over the 3 replicates, and statistical analysis was performed using GraphPad Prism. Clustering of marker phosphorylation profiles was performed in MatLab using a Euclidean metric.

EMD Library Kinase Annotation. Chemical structures of the EMD kinase inhibitor library were standardized using an in-house protocol implemented in Pipeline Pilot (Accelrys). Salts/addends and duplicate fragments were removed (using an in-house salt library) so that each structure consisted of only one fragment. Stereochemistry and charges were standardized; the structures were then ionized at $\mathrm{pH}$ $=7.4$ and tautomers were canonicalized.

The Kinase Knowledge Base (KKB, Eidogen-Sertanty, kinasedata.com, Q4 2009) was used to annotate compounds in the EMD library. Structures and data were processed as previously described..$^{47}$ Briefly, structures were standardized using the same Pipeline Pilot in-house protocol as above. From the assay experiments captured in KKB only data from biochemical assays (enzymatic assays, performed with purified protein) of human species were kept and mutant kinase targets were excluded. Only high-quality concentration-response end points $\left(\right.$ e.g., $\mathrm{IC}_{50}, K_{\mathrm{i}}$ ) were used. Kinase activities were transformed into $p$-values (i.e., $p \mathrm{IC}_{50}=-\log _{10}\left[\mathrm{IC}_{50}\right]$ in molar concentration). Kinase targets were standardized based on Entrez Gene IDs. Data were aggregated (using median) first within each assay protocol (experiment) and then across protocols by unique structures and kinase targets. The aggregated kinase concentration-response activity data were then mapped to the EMD library based on unique chemical structures.

Cortical Slice Culture. Slice cultures were prepared as previously described. ${ }^{44}$ Briefly, a manually operated Mcllwain tissue chopper was used to prepare cross sections ( $350 \mu \mathrm{m})$ of P5 rat cortices comprising both hemispheres connected by the corpus callosum. Slices were mounted on Millicell culture inserts ( $30 \mathrm{~mm}$ Organotypic PTFE 0.4 $\mu \mathrm{m})$ suspended over culture media (50\%, Basal Medium, Eagle's, $25 \%$ Horse Serum Heat Inactivated, 18\% HBSS, 2\% SM1 supplement, 4 $\mathrm{mM}$ L-glutamine, $6 \mathrm{mg} \mathrm{mL}^{-1} \mathrm{D}$-glucose, and Pen/Strep) to allow upward diffusion into the slice. Viral particles containing eGFP recombinant DNA, cloned into a pAAV8-MCS backbone and prepared at the Miami Project Viral Core, were applied to the slices. 
Sections were rinsed $24 \mathrm{~h}$ later to remove remaining virus. After 1 week, slices were transected along the midline and paired with fresh contralateral P5 slices that had not been treated with viral particles. Culture media was refreshed with kinase inhibitor added to a final concentration of $10 \mu \mathrm{M}$ and subsequently refreshed at 2-3 day intervals. Control slices received the equivalent amount of DMSO $(0.1 \% \mathrm{v} / \mathrm{v}$ final concentration). Seven days after pairing and initial treatment, slices were fixed with $4 \%$ paraformaldehyde $/ 4 \%$ sucrose in PBS at RT for 30-45 min, then rinsed with PBS, and mounted onto glass slides. Confocal microscopy was used to image EGFP+ profiles at $1 \mathrm{~mm}$ from the transaction border at multiple Z-planes, which were combined into a single flat image. The automated neurite detection algorithm developed by $\mathrm{Wu}$ et $\mathrm{al}^{45}$ was used to quantify total axon growth into the imaged strip, reported relative to growth in DMSO control.

\section{ASSOCIATED CONTENT}

\section{S Supporting Information}

This material is available free of charge via the Internet at http://pubs.acs.org.

\section{AUTHOR INFORMATION}

\section{Corresponding Author}

*E-mail: vlemmon@med.miami.edu; jbixby@med.miami.edu.

\section{Notes}

The authors declare no competing financial interest.

\section{ACKNOWLEDGMENTS}

We thank current and former members of the LemBix laboratory for assisting with several steps of this project, especially Y. Shi with the HCA, A. Oliva and T. Slepak with the primary cell culture, and Y. Martinez with the slice cultures. We acknowledge resources from the Center for Computational Science. V.P.L. holds the Walter G Ross Distinguished Chair in Developmental Neuroscience. We thank P. Hong at Brandeis University for providing guidance and executables for the neurite extraction software. This work was supported by grants from the James and Esther King Biomedical Research Program JEK09KW-05 (JLB), the U.S Army W81XWH-05-1-0061 (V.P.L., J.L.B.), the National Institutes of Health [HD057521 (V.P.L.), NS059866 (J.L.B.)], and the Buoniconti Fund.

\section{REFERENCES}

(1) Anderson, K. D. (2004) Targeting recovery: priorities of the spinal cord-injured population. J. Neurotrauma 21, 1371-83.

(2) Cafferty, W. B. J., Mcgee, A. W., and Strittmatter, S. M. (2008) Axonal growth therapeutics: regeneration or sprouting or plasticity? Trends Neurosci. 31, 215-20.

(3) Zhang, J., Yang, P. L., and Gray, N. S. (2009) Targeting cancer with small molecule kinase inhibitors. Nat. Rev. Cancer 9, 28-39.

(4) Klebl, B. M., and Müller, G. (2005) Second-generation kinase inhibitors. Expert Opin. Ther. Targets 9, 975-93.

(5) Pearce, L. R., Komander, D., and Alessi, D. R. (2010) The nuts and bolts of AGC protein kinases. Nat. Rev. Mol. Cell Biol. 11, 9-22.

(6) Pettus, L. H., and Wurz, R. P. (2008) Small molecule p38 MAP kinase inhibitors for the treatment of inflammatory diseases: novel structures and developments during 2006-2008. Curr. Top. Med. Chem. 8, 1452-67.

(7) Duffy, P., Schmandke, A., Schmandke, A., Sigworth, J., Narumiya, S., Cafferty, W. B. J., and Strittmatter, S. M. (2009) Rho-associated kinase II (ROCKII) limits axonal growth after trauma within the adult mouse spinal cord. J. Neurosci. 29, 15266-76.

(8) Don, A. S. A., Tsang, C. K., Kazdoba, T. M., D’Arcangelo, G., Young, W., and Zheng, X. F. S. (2012) Targeting mTOR as a novel therapeutic strategy for traumatic CNS injuries. Drug Discovery Today $17,861-8$.
(9) Michaelevski, I., Segal-Ruder, Y., Rozenbaum, M., Medzihradszky, K. F., Shalem, O., Coppola, G., Horn-Saban, S., Ben-Yaakov, K., Dagan, S. Y., Rishal, I., Geschwind, D. H., Pilpel, Y., Burlingame, A. L., and Fainzilber, M. (2010) Signaling to transcription networks in the neuronal retrograde injury response. Sci. Signaling 3, ra53.

(10) Buchser, W. J., Slepak, T. I., Gutierrez-Arenas, O., Bixby, J. L., and Lemmon, V. P. (2010) Kinase/phosphatase overexpression reveals pathways regulating hippocampal neuron morphology. Mol. Syst. Biol. 6, 391.

(11) Park, K. K., Liu, K., Hu, Y., Kanter, J. L., and He, Z. (2010) PTEN/mTOR and axon regeneration. Exp. Neurol. 223, 45-50.

(12) Loh, S. H. Y., Francescut, L., Lingor, P., Bähr, M., and Nicotera, P. (2008) Identification of new kinase clusters required for neurite outgrowth and retraction by a loss-of-function RNA interference screen. Cell Death Differ. 15, 283-98.

(13) Lie, M., Grover, M., and Whitlon, D. S. (2010) Accelerated neurite growth from spiral ganglion neurons exposed to the Rho kinase inhibitor H-1152. Neuroscience 169, 855-62.

(14) Samara, C., Rohde, C. B., Gilleland, C. L., Norton, S., Haggarty, S. J., and Yanik, M. F. (2010) Large-scale in vivo femtosecond laser neurosurgery screen reveals small-molecule enhancer of regeneration. Proc. Natl. Acad. Sci. U.S.A. 107, 18342-7.

(15) Swinney, D. C., and Anthony, J. (2011) How were new medicines discovered? Nat. Rev. Drug Discovery 10, 507-19.

(16) Bain, J., Plater, L., Elliott, M., Shpiro, N., Hastie, C. J., McLauchlan, H., Klevernic, I., Arthur, J. S. C., Alessi, D. R., and Cohen, P. (2007) The selectivity of protein kinase inhibitors: a further update. Biochem. J. 408, 297-315.

(17) Ghoreschi, K., Laurence, A., and Shea, J. J. O. (2009) Selectivity and therapeutic inhibition of kinases: to be or not to be? Nat. Immunol. $10,356-360$.

(18) Zhang, J. J.-H., Chung, T., and Oldenburg, K. (1999) A simple statistical parameter for use in evaluation and validation of high throughput screening assays. J. Biomol. Screening 4, 67-73.

(19) Anastassiadis, T., Deacon, S. W., Devarajan, K., Ma, H., and Peterson, J. R. (2011) Comprehensive assay of kinase catalytic activity reveals features of kinase inhibitor selectivity. Nat. Biotechnol. 29, $1039-45$.

(20) Loudon, R. P., Silver, L. D., Yee, H. F., and Gallo, G. (2006) RhoA-kinase and myosin II are required for the maintenance of growth cone polarity and guidance by nerve growth factor. J. Neurobiol. 66, 847-67.

(21) Gallo, G. (2006) RhoA-kinase coordinates F-actin organization and myosin II activity during semaphorin-3A-induced axon retraction. J. Cell Sci. 119, 3413-23.

(22) Gallo, G., Yee, H. F., and Letourneau, P. C. (2002) Actin turnover is required to prevent axon retraction driven by endogenous actomyosin contractility. J. Cell Biol. 158, 1219-28.

(23) Monnier, P. P., Sierra, A., Schwab, J. M., Henke-Fahle, S., and Mueller, B. K. (2003) The Rho/ROCK pathway mediates neurite growth-inhibitory activity associated with the chondroitin sulfate proteoglycans of the CNS glial scar. Mol. Cell. Neurosci. 22, 319-330.

(24) Minase, T., Ishima, T., Itoh, K., and Hashimoto, K. (2010) Potentiation of nerve growth factor-induced neurite outgrowth by the ROCK inhibitor Y-27632: a possible role of $\mathrm{IP}_{3}$ receptors. Eur. J. Pharmacol. 648, 67-73.

(25) Lingor, P., Teusch, N., Schwarz, K., Mueller, R., Mack, H., Bähr, M., and Mueller, B. K. (2007) Inhibition of Rho kinase (ROCK) increases neurite outgrowth on chondroitin sulphate proteoglycan in vitro and axonal regeneration in the adult optic nerve in vivo. J. Neurochem. 103, 181-9.

(26) Feng, Y., Yin, Y., Weiser, A., Griffin, E., Cameron, M. D., Lin, L., Ruiz, C., Schürer, S. C., Inoue, T., Rao, P. V., Schröter, T., and Lograsso, P. (2008) Discovery of substituted 4-(pyrazol-4-yl)-phenylbenzodioxane-2-carboxamides as potent and highly selective Rho kinase (ROCK-II) inhibitors. J. Med. Chem. 51, 6642-5.

(27) Diez, H., Garrido, J. J., and Wandosell, F. (2012) Specific roles of Akt iso forms in apoptosis and axon growth regulation in neurons. PloS One 7, e32715. 
(28) Park, K. K., Liu, K., Hu, Y., Smith, P. D., Wang, C., Cai, B., Xu, B., Connolly, L., Kramvis, I., Sahin, M., and He, Z. (2008) Promoting axon regeneration in the adult CNS by modulation of the PTEN/ mTOR pathway. Science 322, 963-6.

(29) Abe, N., Borson, S. H., Gambello, M. J., Wang, F., and Cavalli, V. (2010) Mammalian target of rapamycin (mTOR) activation increases axonal growth capacity of injured peripheral nerves. J. Biol. Chem. 285, 28034-43.

(30) Wang, P., Verin, A. D., Birukova, A., Gilbert-McClain, L. I., Jacobs, K., and Garcia, J. G. (2001) Mechanisms of sodium fluorideinduced endothelial cell barrier dysfunction: role of MLC phosphorylation. Am. J. Physiol.: Lung Cell. Mol. Physiol. 281, L1472-83.

(31) Schmidt, J. T., Morgan, P., Dowell, N., and Leu, B. (2002) Myosin light chain phosphorylation and growth cone motility. $J$. Neurobiol. 52, 175-88.

(32) Sivasankaran, R., Pei, J., Wang, K. C., Zhang, Y. P., Shields, C. B., $\mathrm{Xu}, \mathrm{X} . \mathrm{M}$., and He, Z. (2004) PKC mediates inhibitory effects of myelin and chondroitin sulfate proteoglycans on axonal regeneration. Nat. Neurosci. 7, 261-8.

(33) Bixby, J. L. (1989) Protein kinase C is involved in laminin stimulation of neurite outgrowth. Neuron 3, 287-97.

(34) Fischer, M., Pereira, P. M., Holtmann, B., Simon, C. M., Hanauer, A., Heisenberg, M., and Sendtner, M. (2009) P90 Ribosomal s6 kinase 2 negatively regulates axon growth in motoneurons. Mol. Cell. Neurosci. 42, 134-41.

(35) Bixby, J. L., and Jhabvala, P. (1992) Inhibition of tyrosine phosphorylation potentiates substrate-induced neurite growth. J. Neurobiol. 23, 468-80.

(36) Alabed, Y. Z., Pool, M., Ong Tone, S., Sutherland, C., and Fournier, A. E. (2010) GSK3 beta regulates myelin-dependent axon outgrowth inhibition through CRMP4. J. Neurosci. 30, 5635-43.

(37) Gopalakrishnan, S. M., Teusch, N., Imhof, C., Bakker, M. H. M., Schurdak, M., Burns, D. J., and Warrior, U. (2008) Role of Rho kinase pathway in chondroitin sulfate proteoglycan-mediated inhibition of neurite outgrowth in PC12 cells. J. Neurosci. Res. 86, 2214-26.

(38) Gutierrez, H., and Davies, A. M. (2011) Regulation of neural process growth, elaboration and structural plasticity by NF- $\kappa$ B. Trends Neurosci. 34, 316-25.

(39) Yoshimura, K., Ueno, M., Lee, S., Nakamura, Y., Sato, A., Yoshimura, K., Kishima, H., Yoshimine, T., and Yamashimta, T. (2011) C-Jun N-terminal kinase induces axonal degeneration and limits motor recovery after spinal cord injury in mice. Neurosc. Res. 71, 266-77.

(40) Dimitropoulou, A., and Bixby, J. L. (2005) Motor neurite outgrowth is selectively inhibited by cell surface MuSK and agrin. Mol. Cell. Neurosci. 28, 292-302.

(41) Furuya, H., Shimizu, Y., and Kawamori, T. (2011) Sphingolipids in cancer. Cancer Metastasis Rev. 30, 567-76.

(42) Lee, J.-H., Jang, S., Jeong, H.-S., and Park, J.-S. (2011) Effects of sphingosine-1-phosphate on neural differentiation and neurite outgrowth in neuroblastoma cells. Chonnam Med. J. 47, 27-30.

(43) Sato, K., Tomura, H., Igarashi, Y., Ui, M., and Okajima, F. (1997) Exogenous sphingosine 1-phosphate induces neurite retraction possibly through a cell surface receptor in PC12 cells. Biochem. Biophys. Res. Commun. 240, 329-34.

(44) Blackmore, M. G., Wang, Z., Lerch, J. K., Motti, D., Zhang, Y. P., Shields, C. B., Lee, J. K., Goldberg, J. L., Lemmon, V. P., and Bixby, J. L. (2012) Krüppel-like Factor 7 engineered for transcriptional activation promotes axon regeneration in the adult corticospinal tract. Proc. Natl. Acad. Sci. U.S.A. 109, 7517-22.

(45) Wu, C., Schulte, J., Sepp, K. J., Littleton, J. T., and Hong, P. (2010) Automatic robust neurite detection and morphological analysis of neuronal cell cultures in high-content screening. Neuroinformatics 8 , 83-100.

(46) Lerch, J. K., Kuo, F., Motti, D., Morris, R., Bixby, J. L., and Lemmon, V. P. (2012) Isoform diversity and regulation in peripheral and central neurons revealed through RNA-Seq. PloS One 7, e30417.
(47) Schürer, S. C., and Muskal, S. M. (2013) Kinome-wide activity modeling from diverse public high-quality data sets. J. Chem. Inf. Modeling 53, 27-38. 